\title{
Island Growth of Poly(chloro-p-xylylene) Coatings
}

\author{
A. S. Medvedeva ${ }^{a, *}$, D. R. Streltsov ${ }^{a, b}$, P. V. Dmitryakov ${ }^{a}$, A. A. Nesmelov ${ }^{a}$, \\ A. I. Buzin ${ }^{a, b}$, and S. N. Chvalun ${ }^{a, b}$ \\ ${ }^{a}$ National Research Center Kurchatov Institute, Moscow, 123182 Russia \\ ${ }^{b}$ Enikolopov Institute of Synthetic Polymer Materials, Russian Academy of Sciences, Moscow, 117393 Russia \\ *e-mail:medvedeva.as@gmail.com \\ Received March 31, 2020; revised May 26, 2020; accepted July 3, 2020
}

\begin{abstract}
The evolution of the morphology of island poly(chloro- $p$-xylylene) films formed on silicon substrates by vapor deposition polymerization is investigated by atomic force microscopy. The dependences of the effective thickness of the island coating, the number density of polymer islands, and their average size on the surface coverage are studied. The maximal density of polymer islands and the surface coverage corresponding to the transition to the coalescence regime are estimated. Within the framework of the theory of dynamic scaling, the size distribution of islands and the size distribution of their "capture zones" are analyzed. It is shown that, at low degrees of filling of the substrate, before the coalescence of islands, these distributions are described by scaling functions corresponding to the model of reaction-limited aggregation. The size of the critical nucleus is estimated from the size distributions of the "capture zones" of polymer islands.
\end{abstract}

DOI: $10.1134 / \mathrm{S} 156009042006007 \mathrm{X}$

\section{INTRODUCTION}

Poly( $p$-xylylenes) (known by their commecial name parylenes) are linear aromatic polymers formed by vapor deposition polymerization. This method allows one to obtain continuous uniform coatings with controlled thickness without the use of catalysts and solvents [1]. Owing to low gas and moisture permeability, poly ( $p$-xylylenes) are used as protective coatings in microelectronics and medicine [2]. Compared to unsubstituted poly ( $p$-xylylene) (PPX), poly(chloro- $p$ xylylene) (PCPX) has higher barrier characteristics (moisture permeability at $37^{\circ} \mathrm{C}$ is $0.0012 \mathrm{ng} /(\mathrm{Pa} \mathrm{s} \mathrm{m})$ for PPX and $0.0004 \mathrm{ng} /(\mathrm{Pa} \mathrm{s} \mathrm{m})$ for PCPX) [1] and a higher glass transition temperature $\left(13^{\circ} \mathrm{C}\right.$ for PPX [1] and about $84^{\circ} \mathrm{C}$ for PCPX [3]). Furthermore, the advantages of PCPX are a higher growth rate of the coating at room temperature and a high ceiling deposition temperature $\left(30^{\circ} \mathrm{C}\right.$ for $\mathrm{PPX}$ and $90^{\circ} \mathrm{C}$ for PCPX) [4]. Owing to its resistance against aggressive biological media, PCPX is used in medicine, for example, in the production of protective coatings for biosensors [5] and insulating layers in microelectromechanical systems for biomedical purposes [6]. At the same time, despite the great practical interest in thin-film coatings based on PPX, there are a number of problems that remain unsolved.

It is known that the surface morphology of thin films and, consequently, their optical, hydrophobic, and tribological properties are largely determined by the initial stages of film formation. For inorganic (metal, semiconductor, oxide) island films, a large number of experimental and theoretical works devoted to the kinetic study of their growth have been published [7-9]. Scaling relations have been derived and experimentally confirmed that make it possible to estimate such important kinetic parameters as the size of the critical nucleus, the diffusion coefficient of adatoms (adsorbed atoms) over the substrate, the energy of interaction of atoms in a cluster, and the value of the energy barrier to the transport of adatoms between layers in the case of multilayer growth from the dependence of the number island density on the flow of atoms from the gas phase, the surface coverage, and the deposition temperature. Critical island size $i$ measured in the number of atoms or molecules is an important parameter that determines the process of nucleation of new islands. The island of size $i$ or less for given values of the substrate temperature $T$ and monomer flow from the gas phase $F$ can with a high probability disintegrate into the original atoms or molecules. On the contrary, islands of size $i+1$ or more grow with greater probability than decay. To date, several methods have been developed for assessing the critical island size $i$ from experimental data on the growth of island films. The first one was suggested by J.A. Venables back in the 1970s [10, 11]. This method is based on the analysis of the dependence of the number island density $N_{\text {isl }}$ on the monomer flow from the gas phase $F$ at constant substrate temperature $T$ and the surface coverage $\theta$. The second method was developed in the 1990s [12]. It is based on the approximation of the size distribution of islands by some general 
scaling function, the form of which is determined by critical island size $i$. It should be noted that, if in the first method to determine $i$ a series of samples should be formed at different deposition rates, then in the second case basically one sample is sufficient. Both approaches have been successfully used to analyze the growth of island films of metal and semiconductor coatings.

Another approach to estimating the critical island size $i$, which has been actively developing in recent years, is based on an analysis of the size distribution not of the islands themselves but of the areas of their "capture zones." Since the island grows owing to the attachment of adatoms deposited near it, it seems natural to have the idea to separate the entire surface of the substrate so that the island is inside a certain region from which atoms or molecules are attached to it. It is assumed that the monomer adsorbed into the "capture zone" of a given island is most likely to attach to it. Thus, the area of the "capture zone" determines the growth rate of the polymer island. The simplest division is tesellate the substrate surface into Voronoi polygons associated with the centers of mass for each island. It was shown that for compact islands such a tesellation makes it possible to estimate their "capture zones" with sufficient accuracy [13]. It is important to note that the analytical expression making it possible to approximate the size distribution of the "capture zones" and to determine critical island size $i$ was obtained not only for the case of diffusion-limited aggregation but also for the case of reaction-limited aggregation. This approach was successfully used to analyze the growth of island films based on organic semiconductors [14, 15].

The problem of describing the kinetics of the growth of organic films on inorganic substrates, which has received much attention in recent years in connection with the development of molecular electronics and molecular photovoltaics, is more complicated than the description of the growth of inorganic films. It is important here which of the concepts developed to describe the growth of metal and semiconductor films remain valid and which require modification owing to their large size, anisotropic shape, and internal degrees of freedom of organic molecules that are much more complex in their structure. In particular, it was found that the diffusion-limited aggregation (DLA) approximation, which implies the instant attachment of an atom or molecule to the growing island upon contact, is sometimes inapplicable. In this case, it is necessary to introduce into the description of the kinetics of island film growth an additional energy parameter that determines the probability of attachment, i.e., to switch from the model of diffusion-limited aggregation to the model of reaction-limited aggregation (RLA).
The growth of polymer films synthesized from the gas phase also has a number of significant features. Important factors here are both the polymer nature of the coating and, therefore, a limited number of growth centers (the adsorbed monomer can attach only to the ends of growing polymer chains), relaxation of polymer chains, their anisotropy, and the dependence of molecular mobility on the molecular weight of the growing chain and a higher permeability of the polymer film for monomer, especially at temperatures above the glass transition temperature of the polymer. In $[16,17]$, when trying to use the scaling approach [18] to analyze the growth of island poly(chloro- $p$ xylylene) coatings, the authors found a number of significant features. The scaling coefficients for the dependences of the number density of polymer islands of the substrate and the average radius of the island on the surface coverage differed from the values obtained within the DLA model. In addition, with an increase in the surface coverage, the shape of the island size distribution changed from a unimodal aggregation mode characteristic of DLA $[12,18]$ to a monotonically decreasing one. The authors suggested that the observed differences can be explained by the processes of relaxation of polymer chains and the formation of closed polymer loops, which reduces the number of polymer chain ends accessible to the monomer and leads to an increase in the monomer concentration on the surface and, hence, in the nucleation rate. The transition from the unimodal bell-shaped size distribution of islands, which is characteristic of the diffusion-limited aggregation, to the monotonically decreasing one in the case of reaction-limited aggregation is described in the theoretical article [19]. These authors took into account the reversibility of the attachment of adatoms to the island by introducing the energy barrier to attachment, an increase in which corresponds to the transition from DLA to RLA aggregation.

In our previous work [20] devoted to the study of the evolution of the morphology of poly ( $p$-xylylene) island films on silicon substrates at deposition temperatures of $23^{\circ} \mathrm{C}$ and $0^{\circ} \mathrm{C}$, there were also differences from the diffusion-limited aggregation model, namely, an increase in the number island density of polymer islands with increasing substrate temperature and the asymmetric shape of the island size distribution. These features were explained by the reversibility of the addition of the monomer to the polymer island.

The aim of this work is to study the island stage of the growth of poly(chloro- $p$-xylylene) coatings until formation of a continuous polymer coating at a fixed monomer flow $F$ using scanning probe microscopy. The dependences of the effective coating thickness $d_{\text {eff }}$ and the number of islands per unit surface area of the substrate $N_{\text {isl }}$ on the surface coverage $\theta$ are investigated. The evolution of the average size of the islands 
$\bar{R}$, the size distribution of polymer islands, and the size distribution of their "capture zones" are analyzed.

\section{EXPERIMENTAL}

Island coatings were formed by vapor deposition polymerization (the Gorham method) [21] on the surface of silicon wafers. Before applying PCPX coatings, the substrates were purified according to the RCA method (Radio Corporation of America) in a solution consisting of five parts of water, one part of hydrogen peroxide, and 0.5 parts of ammonia [22] at a temperature of $75-80^{\circ} \mathrm{C}$. After $10-20 \mathrm{~min}$, the substrates were removed from the solution and washed with deionized water with a specific conductivity of $\leq 10^{-4} \mathrm{~S} / \mathrm{cm}$ and total organic carbon content $\leq 500 \mathrm{ppb}$ which was prepared on a Millipore Milli Q setup (Merck KGaA, Germany). Excess water was removed by blowing an argon flow over the substrates.

The vacuum unit for the formation of PCPX coatings consisted of a sublimation zone, a pyrolysis zone, and a deposition reactor, where a substrate holder was located. During the formation of coatings, vacuum in the unit was maintained at a level of $10^{-5}-10^{-6} \mathrm{~mm} \mathrm{Hg}$. The cyclic dimer of chloro- $p$-xylylene dichloro-[2, 2]paracyclophane (Specialty Coating Systems, Inc., United States) was sublimated at a fixed temperature of $60^{\circ} \mathrm{C}$; then its vapor passed through the pyrolysis zone at a temperature of $650^{\circ} \mathrm{C}$, in which the dimer decomposed to form a reactive monomer chloro- $p$ xylylene. The monomer was condensed on a substrate, the temperature of which was maintained at $25^{\circ} \mathrm{C}$, and polymerized. The surface coverage $\theta$ (the fraction of the substrate surface occupied by polymer islands) was varied by changing the deposition time at a constant monomer flow $F$ set by the temperature of sublimation of the cyclic dimer. The unit was equipped with a shutter, which prevented the contamination of the substrates with low molecular weight products sublimated during heating of the pyrolysis zone and made it possible to more accurately control the monomer deposition time at a constant flow.

The morphology of the samples was studied by AFM using a Multimode 8 microscope with a Nanoscope V controller (Bruker Nano Inc., United States) in the tapping mode in air at room temperature. The probes were RTESPA-300 silicon cantilevers (Bruker Nano Inc., United States) with a nominal resonance frequency of $\sim 300 \mathrm{kHz}$, a force constant of $\sim 40 \mathrm{~N} / \mathrm{m}$, and a probe radius of curvature of $\sim 8 \mathrm{~nm}$. The size of the scan area was $2 \times 2 \mu \mathrm{m}$ with a resolution of $1024 \times$ 1024 points. To collect the necessary statistics, several AFM images were taken for each sample and from 3000 to 10000 polymer islands were analyzed depending on the deposition time.
AFM images were analyzed using the Gwyddion program [23]. To determine the effective thickness of the island polymer coating $d_{\text {eff }}$, for all AFM images obtained for a given sample, histograms of the height distribution were plotted. At the initial stages of growth at low surface coverages $\theta$, the main contribution is made by the randomly rough relief of the substrate and the height distribution itself is close to normal. As the coverage increases, the height distribution becomes bimodal since a peak corresponding to polymer islands appears. To evaluate $d_{\text {eff }}$, the peak corresponding to the roughness of the silicon substrate not occupied by the polymer was subtracted from the height distribution. Then, from the average value of the obtained difference, the average level of the substrate was subtracted and multiplied by the surface coverage $\theta$. For each sample, the effective coating thickness was averaged over several AFM images.

Further image processing was performed using scripts written in the Matlab software environment (The MathWorks, Inc.) by the method described in [20]. The islands touching the image boundaries were excluded from the analysis because of the impossibility of assessing their size. Objects with an area less than 10 pixels were also excluded from the analysis, which approximately corresponds to an island radius of $3.5 \mathrm{~nm}$. For islands detected in the images, the coordinates of their center, the equivalent radius $R$, and the surface coverage $\theta$ were determined.

The critical island size $i$ was estimated using two approaches. In one of them, the size distribution of islands was analyzed. According to the theory of dynamic scaling of island film growth, in the stationary mode of aggregation before coalescence, when the average size of islands $\bar{R}$ is the only characteristic size in the system, the size distribution of islands $A$ at the surface coverage $\theta$ is described by the relation $N_{s}(\theta)=\theta \bar{R}^{-2} f(u)$, where $\bar{R}$ is the average size of the islands, $u=A / \bar{A}$ is the area of the island divided by the average value for all islands, and $f(u)$ is a dimensionless scaling function. It was shown [12] that, within the framework of the diffusion-limited aggregation model, function $f(u)$ can be approximated by an expression that allows calculating critical island size $i$ :

$$
f_{i}(u)=C_{i} u^{i} e^{-i a_{i} u^{\frac{1}{a_{i}}}},
$$

where constants $C_{i}$ and $a_{i}$ are related by equations

$$
\frac{\Gamma\left[(i+2) a_{i}\right]}{\Gamma\left[(i+1) a_{i}\right]}=\left(i a_{i}\right)^{a_{i}}
$$




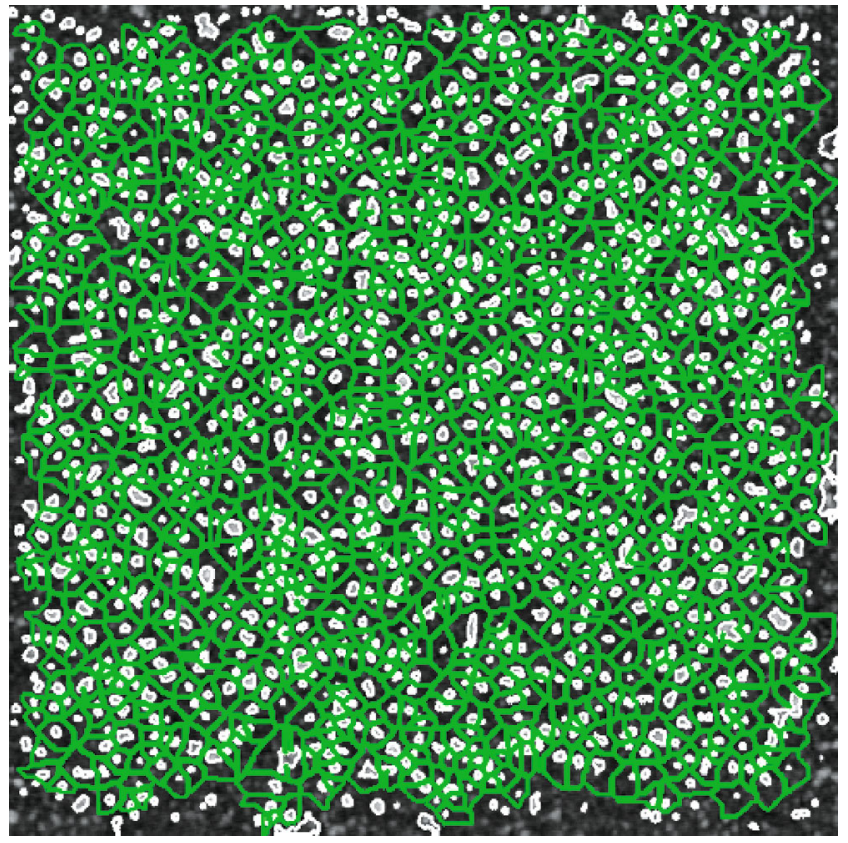

Fig. 1. AFM image of the island poly(chloro- $p$-xylylene) coating formed on the silicon substrate with highlighted polymer islands. Tesselation of the image into Voronoi polygons approximating the "capture zones" of each island is shown. The polygons touching the boundaries of the image are excluded. The image size is $2 \times 2 \mu \mathrm{m}^{2}$. Color drawings can be viewed in the electronic version.

$$
C_{i}=\frac{\left(i a_{i}\right)^{(i+1) a_{i}}}{a_{i} \Gamma\left[(i+1) a_{i}\right]}
$$

ensuring the fulfillment of the normalization conditions $\int_{0}^{\infty} f_{i}(u) d u=\int_{0}^{\infty} f_{i}(u) u d u=1$.

Within the framework of another approach, which has become widespread recently, not the size distribution of islands but the size distribution of their "capture zones" is analyzed. In accordance with [13], for compact islands, the "capture zone" for each island can be approximated with a sufficient accuracy by dividing the substrate surface into Voronoi polygons associated with the centers of mass of the islands. The Voronoi polygon is a convex polygon, each point in which is closer to the center of the island in it than to the centers of all the others (Fig. 1).

It should be noted that, for the same AFM image, when constructing the size distribution of islands, a slightly larger number of objects are analyzed than when analyzing their "capture zones." This is because the "capture zones" that touch the image boundaries should be excluded from the analysis, while the corresponding polymer islands that do not touch the image boundaries are included in the size distribution analysis (Fig. 1).
As was shown in [13], the size distribution function of "capture zones" can be approximated by the generalized Wigner distribution

$$
P_{\beta}(s)=\alpha_{\beta} s^{\beta} \exp \left(-b_{\beta} s^{2}\right),
$$

where $s$ is the area of the "capture zone" divided by its average value and the coefficients $a_{\beta}$ and $b_{\beta}$ depend on the parameter $\beta$, which is related to critical island size $i$ $(\beta=i+2$ for the DLA model and $\beta=(i+3) / 2$ for the RLA model):

$$
\begin{gathered}
\alpha_{\beta}=2 \Gamma\left(\frac{\beta+2}{2}\right)^{\beta+1} / \Gamma\left(\frac{\beta+1}{2}\right)^{\beta+2}, \\
b_{\beta}=\left[\Gamma\left(\frac{\beta+2}{2}\right) / \Gamma\left(\frac{\beta+1}{2}\right)\right]^{2} .
\end{gathered}
$$

Thus, using the function of the size distribution of the areas of "capture zones" of islands, one can determine the parameter $\beta$ from which the critical island size $i$ can be derived within both the DLA and RLA aggregation models.

\section{RESULTS AND DISCUSSION}

Figure 2 shows the AFM images of PCPX island films with the surface coverages from 2 to $35 \%$.

The islands, as in the case of unsubstituted PPX [20], have a rounded shape and a compact boundary. As was noted in [24], island polymer films obtained by vapor deposition are characterized by two classes of morphology: islands with compact and smooth boundaries and islands with a fractal morphology. The realization of one of these morphologies is determined by the ratio of the characteristic times of surface diffusion of the monomer adsorbed on the substrate and the relaxation of macromolecules. The shape of PCPX islands differs significantly from the fractal one typical of diffusion-limited aggregation. A similar conclusion was reached by the authors of [16, 17], who also studied the island growth of PCPX films. They suggested that the compact shape of the islands is due to the processes of relaxation of polymer chains and strong intermolecular interactions.

The growth rate of the effective film thickness at a sublimation temperature of $60^{\circ} \mathrm{C}$ and a substrate temperature of $25^{\circ} \mathrm{C}$ is about $0.03 \mathrm{~nm} / \mathrm{min}$, which is approximately three orders of magnitude less than that in [16], where this value was $20-30 \mathrm{~nm} / \mathrm{min}$ at room temperature of the substrate and a sublimation temperature of $120^{\circ} \mathrm{C}$. At surface coverages less than $\theta_{c} \sim 0.25$ (onset of the coalescence of islands), the number island density $N_{\text {isl }}$ grows according to the power law $N_{\text {isl }} \sim \theta^{q}$, reaching a maximum value of $460 \pm 60 \mu^{-2}$ (Fig. 3a). At higher coverages, $N_{\text {isl }}$ begins to decrease. It should be noted that a decrease in the monomer flow led not only to a slowdown in film growth as compared to [16] but also to a 


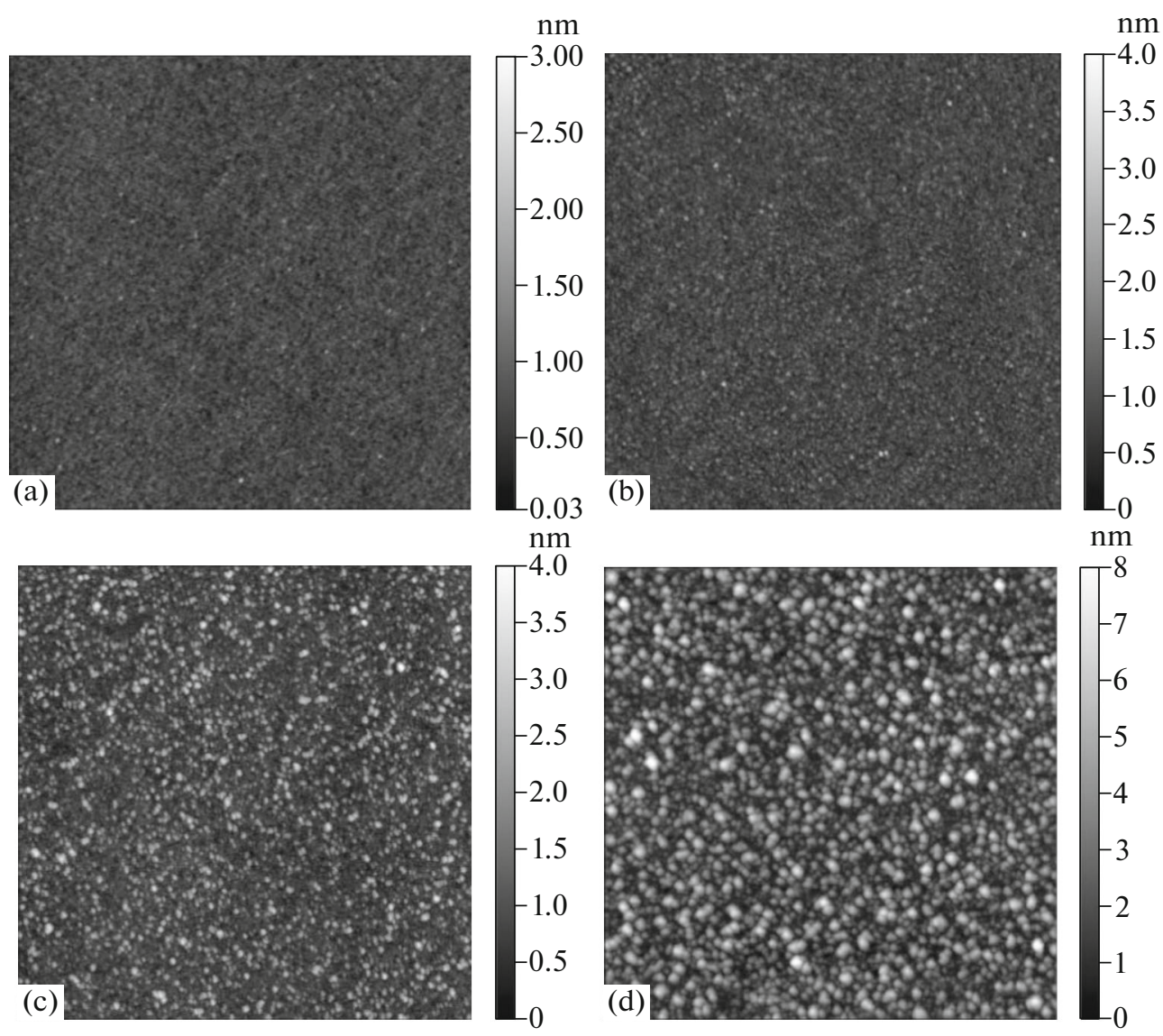

Fig. 2. AFM images of island PCPX coatings obtained on silicon wafers at different times of monomer deposition and at a sublimation temperature of $60^{\circ} \mathrm{C}$ and a substrate temperature of $25^{\circ} \mathrm{C}$. Deposition time: (a) 1, (b) 3, (c) 7, and (d) 30 min; effective coating thickness $d_{\text {eff: }}$ (a) 0.01 , (b) 0.1 , (c) 0.16 , and (d) $0.8 \mathrm{~nm}$; surface coverage $\theta$ : (a) 0.02 , (b) 0.05 , (c) 0.19 , and (d) 0.35 . Size of all images is $2 \times 2 \mu \mathrm{m}^{2}$.

lower value of $\theta_{\mathrm{c}}(\sim 0.25)$, while in [16] $\theta_{\mathrm{c}}$ was $\sim 0.35$ (Fig. 3a). The maximal density of islands $N_{\text {isl }}$ amounted to $460 \pm 60 \mu \mathrm{m}^{-2}$, while in [16] this value was $\sim 800 \mu \mathrm{m}^{-2}$.

In the case of diffusion-limited aggregation at constant monomer flow $F$ and diffusion coefficient $D$ in the steady-state growth mode up to the coalescence stage, the density of islands $N_{\text {isl }}$ and the surface coverage $\theta$ are related by the scaling relation $N_{i s l} \sim \theta^{q}$, where $q=1 /(i+2)$ and $i$ is the size of the critical nucleus $[25,26]$. The process of attachment of the monomer to the island (or to the end of a polymer chain) is considered as irreversible with the probability equal to one. The presence of the energy barrier to attachment to the island (transition from the DLA to RLA aggregation mode) leads to the dependence of the exponent $q$ on barrier size and to the increase in $q$ compared to the DLA aggregation mode [25, 27].

In our case, $q=0.30 \pm 0.05$ (Fig. 3a) is between $q=0.33$ and 0.25 corresponding to $i=1$ and 2 in the diffusion-limited aggregation model. In [16], the scal- ing exponent $q$ for the growth of island PCPX coatings at a significantly higher monomer flow from the gas phase was determined as $q=0.23 \pm 0.04$, which is close to our value.

Figure $3 \mathrm{~b}$ shows the dependence of the average radius of the island $\bar{R}$ on the surface coverage $\theta$. At low surface coverages, $\bar{R} \sim \theta^{n}$, where $n=0.34 \pm 0.03$. Upon reaching $\theta_{\mathrm{c}} \sim 0.25$, islands begin to merge, which manifests itself in the form of a characteristic break on the plot, and the exponent of the power dependence $n$ in the scaling ratio $\bar{R} \sim \theta^{n}$ increases to $0.83 \pm 0.12$. Scaling exponents $q$ and $n$, which determine the dependence of the density of islands and their average radius on the surface coverage, are related by the relation $q+n d=1$, where $d=2$ for the growth of two-dimensional islands and $d=3$ for the growth of three-dimensional islands, respectively [28]. In our case, $q+2 n=0.98 \pm 0.06$, which indicates a predominantly two-dimensional growth of islands prior to their coalescence. 

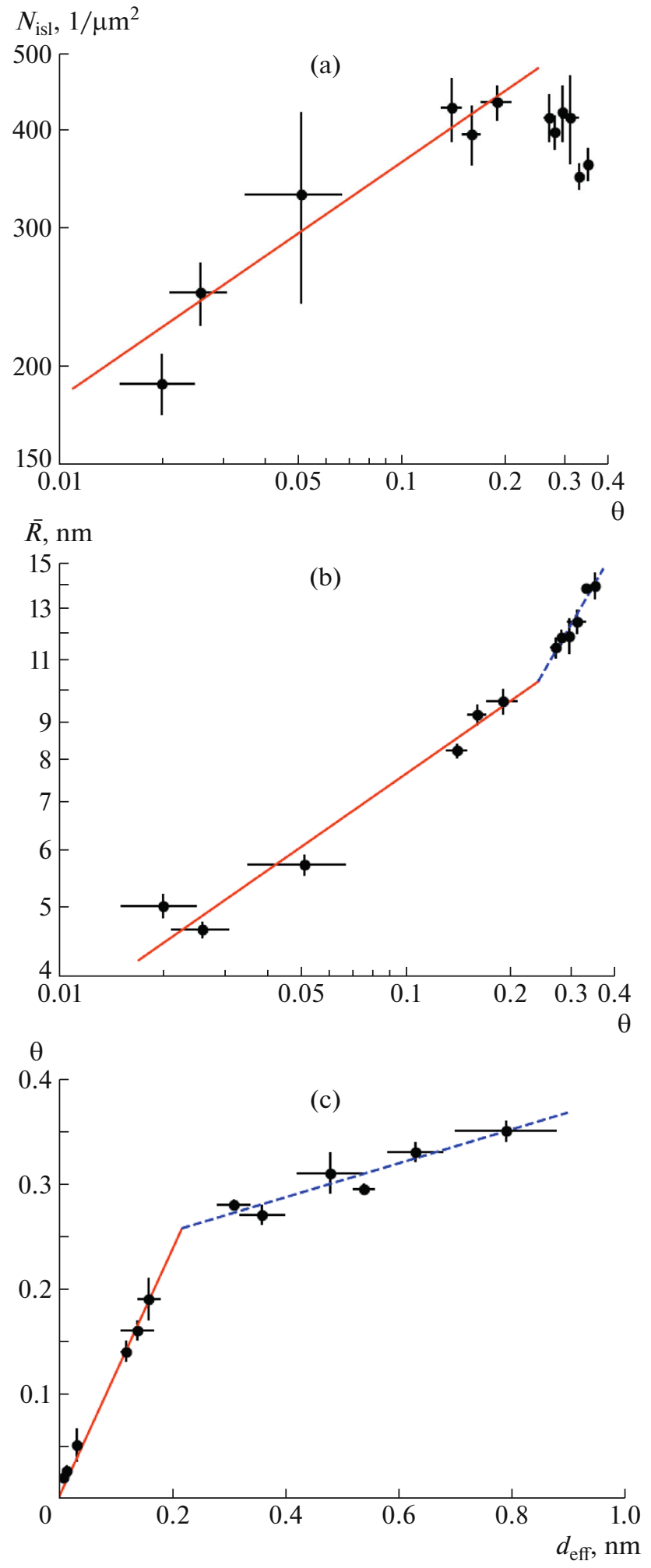

Fig. 3. Dependences of the number of islands $N_{\text {isl }}$ per (a) square micrometer of substrate surface and (b) average island radius $\bar{R}$ on the surface coverage $\theta$ and (c) the dependence of $\theta$ on the effective film thickness $d_{\text {eff }}$. Points refer to experiments, and straight lines refer to $(a, b)$ power-law and (c) linear data approximations. The slope of the straight line: (a) $0.30 \pm 0.05$, (b) $0.34 \pm 0.03$ and $0.83 \pm 0.12$, and (c) $1.18 \pm 0.04$ and $0.16 \pm 0.03 \mathrm{~nm}^{-1}$.
Figure $3 \mathrm{c}$ shows the dependence of surface coverage $\theta$ on the effective film thickness. Upon reaching the effective film thickness $d_{\text {eff }} \sim 0.2 \mathrm{~nm}$, which corresponds to $\theta_{\mathrm{c}} \sim 0.25$, the dependence $\theta$ on the effective film thickness undergoes a break-the slope of the dependence $\theta\left(d_{\text {eff. }}\right)$ decreases by a factor of $\sim 7$. This indicates that the growth rate of island films in the vertical direction begins to prevail over the growth rate in the lateral direction. A similar effect described in [16] is interpreted by the authors by the predominant filling of the areas between the contacting polymer islands, that is, transition to three-dimensional growth of the film, when growth occurs mainly not owing to the diffusion of adsorbed monomer molecules over the substrate but owing to the flow of molecules onto the growing film from the gas phase.

Let us now consider the size distribution of polymer islands, which is mainly influenced by the aggregation mechanism $[12,19,29]$. In the case of diffusion-limited aggregation, the island size distribution can be approximated using Eq. (1). The scaling function of island size distribution $f(s)$ is symmetric, and its maximum is at $s=1[12,18]$. In [27], where the Monte Carlo method was used to simulate the effect of aggregation reversibility on the size distribution, it was shown that an increase in the energy barrier to the attachment of the monomer to the island (i.e., the transition from DLA to RLA) leads to a shift in the peak of the size distribution of islands toward smaller sizes. The shape of the distribution changes from bellshaped to monotonically decreasing. This change can be qualitatively explained as follows: an increase in the energy barrier to the attachment of the monomer to the island leads to a significant slowdown in the island growth rate. With an increase in the energy barrier, the concentration of the monomer on the substrate increases (the monomer flow from the gas phase remains constant) and, as a consequence, the rate of nucleation of new islands grows. All this leads to the formation of a large number of small islands.

As seen in Fig. 4a, at surface coverages (up to $\theta_{c}=$ 0.25 ), the size distribution of the islands is bell-shaped with a maximum at $s \sim 0.3$. Displacement of the distribution maximum relative to $s=1$, which is characteristic of diffusion-limited aggregation, toward smaller island sizes was also reported for unsubstituted PPX [20]. A similar size distribution was also observed in [17] at degrees of substrate filling up to 0.12. As the surface coverage increased, the size distribution of islands [17] became monotonically decreasing in accordance with the reversible aggregation model proposed in [19].

In recent years, in addition to analyzing the size distribution of islands, the size distribution of the areas of "capture zones" of islands has been analyzed in a number of works, both experimental and theoretical $[26,30,31]$. Figure 5 shows histograms of the size distribution of the areas of "capture zones" $P_{\beta}(s)$, where 

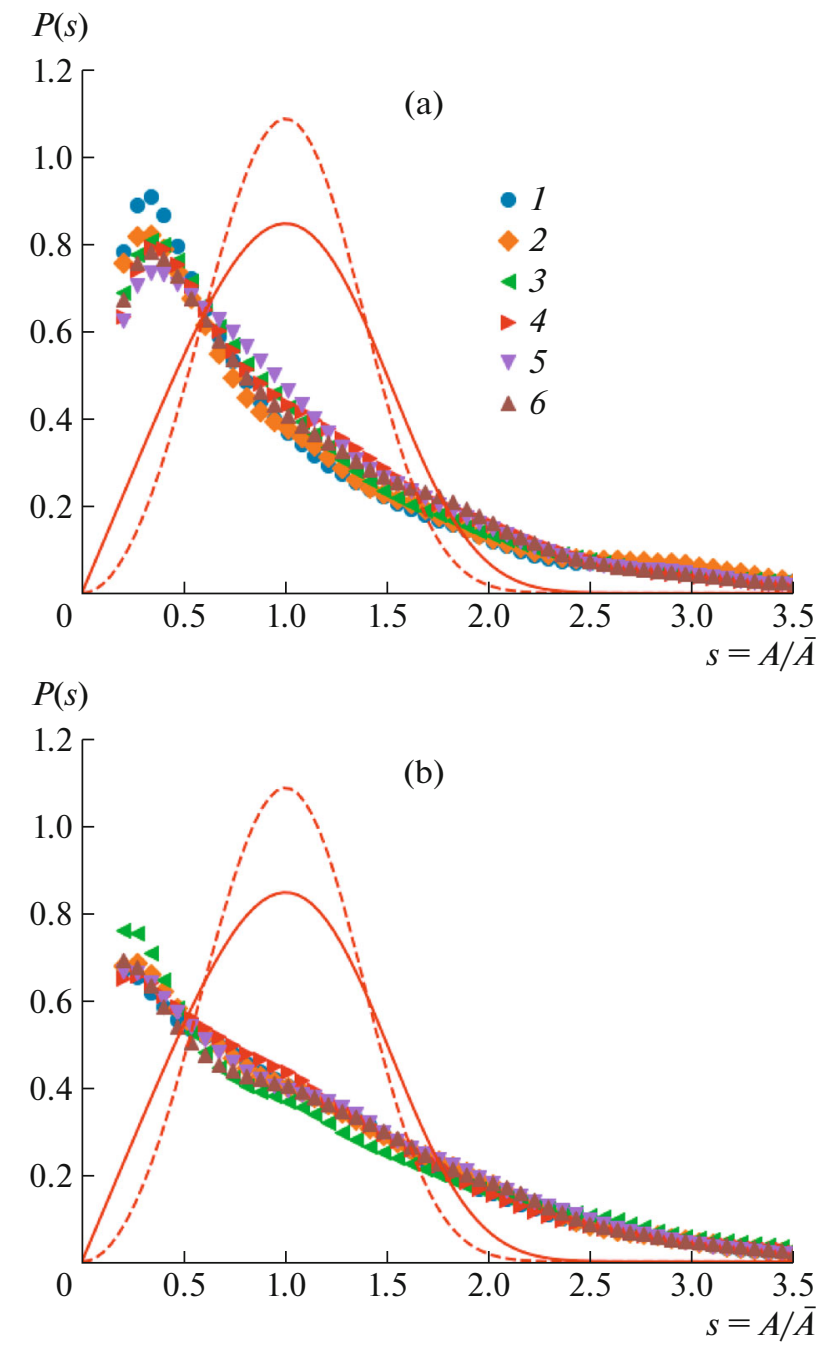

Fig. 4. Functions of island size distribution $P(s)$ with the surface coverage $\theta$ (a) below $\theta_{\mathrm{c}}=0.25$ and (b) above $\theta_{\mathrm{c}}=$ 0.25 . Solid and dotted lines are scaling function $f(s)$ (Eq. (1)) for the values of the critical size of the island $i=$ 1 and 2, respectively; (a) $\theta=$ (1) $2.0 \pm 0.5 \%$, (2) $2.6 \pm$ $0.5 \%$, (3) $5.1 \pm 1.6 \%$, (4) $14 \pm 1 \%$, (5) $16 \pm 1 \%$, and (6) $19 \pm 2 \%$; (b) $\theta=$ (1) $27 \pm 1 \%$, (2) $28 \pm 0.5 \%$, (3) $30 \pm$ $0.5 \%$, (4) $31 \pm 2 \%$, (5) $33 \pm 1 \%$, and (6) $35 \pm 1 \%$.

$s$ is the area of the "capture zone" divided by its average value for samples with the coverage $\theta$ below (Fig. 5a) and above (Fig. 5b) $\theta_{c}=0.25$, corresponding to the onset of coalescence of islands. It can be seen that, in both series, within the statistical scatter, the histograms coincide. It should be noted that, for samples with the coverage below $\theta_{c}=0.25$, the maximum of the distribution is shifted to the left relative to the maximum of the plots of the generalized Wigner distribution (Eq. (4)) for $\beta=1,2$, and 3, which are also shown in Fig. 5a. The experimental histograms are closest to the curve defined by the generalized Wigner distribution with $\beta=2$, which corresponds to the size of the critical nucleus $i=1$ in the reaction-limited aggregation model. This estimate coincides with the value of the critical size of the nucleus for unsubstituted poly ( $p$-xylylene) obtained in [20] and is consistent with the second order of the reaction of the $p$-xylylene polymerization initiation with respect to the monomer derived from quantum chemical calculations [32]. For samples with the coverages $\theta>\theta_{c}$ the distribution becomes even more asymmetric and its maximum shifts to the left, which can be explained by the coalescence of islands, which leads to an increase in the average area of the "capture zone" and, as a consequence, to a shift of the distribution maximum toward smaller sizes.

Asymmetric distribution $P_{\beta}(s)$ and its differences from the generalized Wigner distribution can be explained by the reversibility of the attachment of the monomer to the growing polymer island. For example, it was shown [25] that the introduction of the energy barrier to the attachment of the monomer to the island leads to a decrease in the maximum of the distribution $P_{\beta}(s)$ and its shift toward lower values of $s$. In this case, the size distribution of islands is described not by the generalized Wigner distribution but by the gamma distribution:

$$
P_{\Gamma}(s)=\frac{\alpha^{\alpha}}{\Gamma(\alpha)} s^{\alpha-1} e^{-\alpha s}
$$

As an example, Fig. $5 \mathrm{c}$ shows the histogram of the size distribution of "capture zones" for the sample with the surface coverage $\theta=0.16$. It can be seen that the gamma distribution approximates the histogram much better than the generalized Wigner distribution.

Thus, it can be concluded that the size distributions of PCPX islands and the distribution of their "capture zones" cannot be fully described within the framework of classical models based on diffusion-limited aggregation but can be explained by the reversibility of monomer attachment to the growing polymer island, which is due to the polymer nature of the formed island coatings.

\section{CONCLUSIONS}

In this work, the evolution of the morphology of poly(chloro- $p$-xylylene) island films formed on silicon substrates by vapor deposition polymerization at a deposition temperature of $25^{\circ} \mathrm{C}$ and the fixed monomer flow is investigated. The dependences of the effective thickness of the island coating, the number of polymer islands per unit surface area of the substrate, and their average size on the surface coverage are studied. The maximum density of polymer islands $\left(N_{\mathrm{isl}}=\right.$

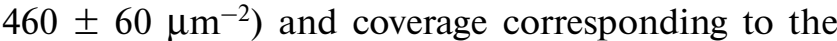
transition to the coalescence regime $\left(\theta_{c}=0.25\right)$ are estimated. The scaling coefficients relating the island 

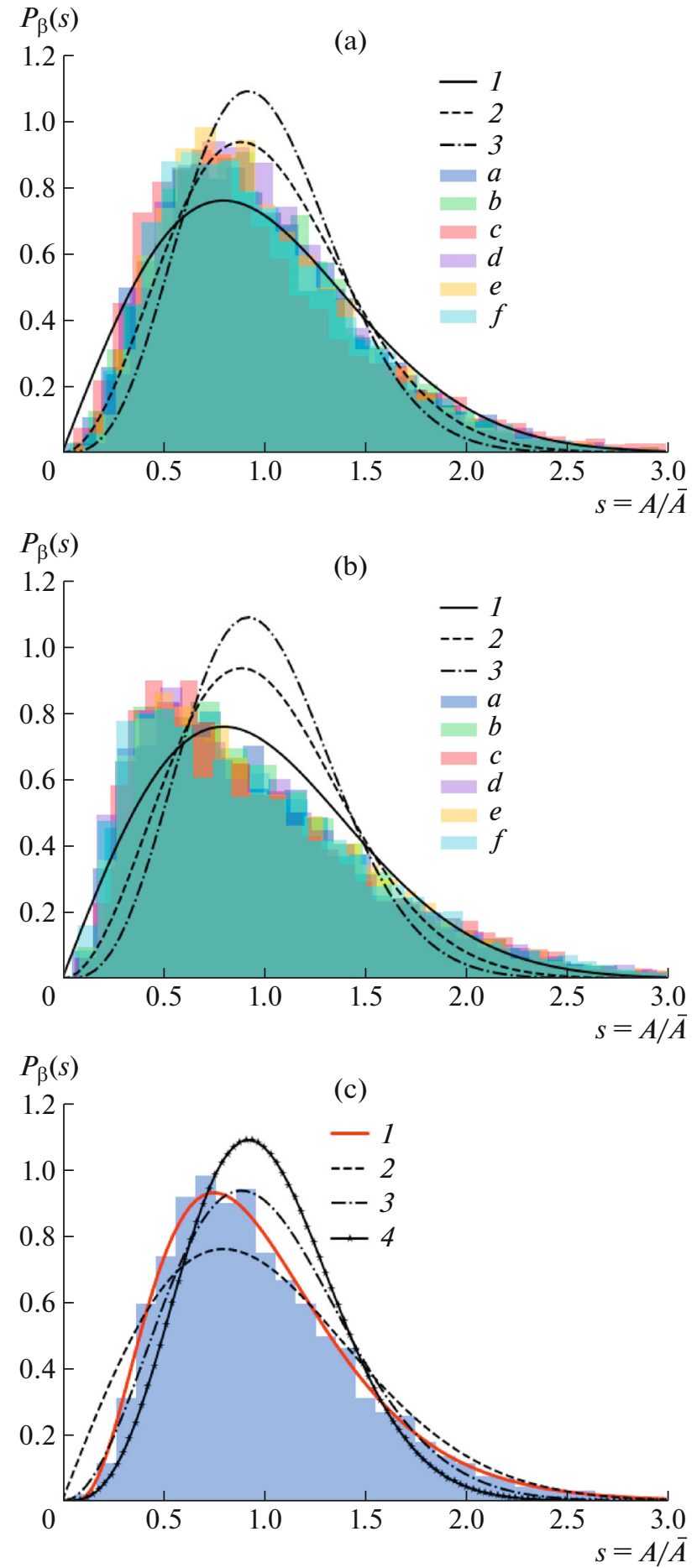

Fig. 5. Histograms of the size distribution of the areas of the "capture zones" of the islands $P_{\beta}(s)$ for a number of sufface coverages $\theta$ : (a) below $\theta_{\mathrm{c}}=0.25$, (b) above $\theta_{\mathrm{c}}=$ 0.25 , and (c) $\theta=0.16$. Curves $1-3$ are the generalized Wigner distributions (Eq. (4)) for $\beta=1,2$, and 3, respectively; curve 4 is the gamma distribution (Eq. (7)). (a) $\theta=$ (a) $2.0 \pm 0.5 \%$, (b) $2.6 \pm 0.5 \%$, (c) $5.1 \pm 1.6 \%$, (d) $14 \pm 1 \%$, (e) $16 \pm 1 \%$, and (f) $19 \pm 2 \%$ and (b) $\theta=$ (a) $27 \pm 1 \%$, (b) $28 \pm 0.5 \%$, (c) $30 \pm 0.5 \%$, (d) $31 \pm 2 \%$, (e) $33 \pm 1 \%$, and $(f) 35 \pm 1 \%$. Coefficient of determination of histogram approximation $R^{2}$ for $\theta=0.16$ is (1) 0.950 , (2) 0.951 , (3) 0.827 , and (4) 0.997 . number density $N_{\text {isl }}$, their average radius $\bar{R}$, and the surface coverage $\theta\left(N_{\text {isl }} \sim \theta^{q}, q=0.30 \pm 0.05\right.$; $\left.\bar{R} \sim \theta^{n}, n=0.34 \pm 0.03\right)$ at the degrees of filling of the substrate $\theta<\theta_{\mathrm{c}}$ are determined.

In addition, the evolution of the size distribution of polymer islands and the size distribution of their "capture zones" is analyzed. It is shown that, at low surface coverage, the island size distributions are described using the same scaling function; however, at sufface coverages corresponding to the transition to the coalescence regime, these scaling relations are violated. The size distributions of the islands and their "capture zones" differ from the theoretical distributions corresponding to the diffusion-limited aggregation models, and their characteristic shape indicates a possible significant effect of the reversibility of the attachment of the adsorbed monomer to the growing polymer island on the growth of island poly(chloro- $p$ xylylene) coatings. The size distributions of the "capture zones" of polymer islands are used to estimate the size of the critical nucleus within the framework of the reaction-limited aggregation model; the critical nucleus size is $i=1$.

\section{ACKNOWLEDGMENTS}

AFM measurements were carried out on the equipment of the Probe and Electron Microscopy Resource Center of the National Research Center Kurchatov Institute.

\section{FUNDING}

This study was supported by the Russian Foundation for Basic Research (project nos. 18-33-00600-a and 18-0000427-a).

\section{OPEN ACCESS}

This article is distributed under the terms of the Creative Commons Attribution 4. International license (http://creativecommons.org/licenses/by/4.0/), which permits unrestricted use, distribution, and reproduction in any medium, provided you give appropriate credit to the original author(s) and the source, provide a link to the Creative Commons license, and indicate if changes were made.

\section{REFERENCES}

1. W. F. Beach, in Kirk-Othmer Encyclopedia of Chemical Technology (Wiley, New York, 2000), p. 587.

2. CVD Polymers: Fabrication of Organic Surfaces and Devices, Ed. by K. K. Gleason (Wiley, New York, 2015).

3. A. Kahouli, F. Jomni, A. Sylvestre, B. Yangui, and J. Legrand, J. Phys. D: Appl. Phys. 44, 505302 (2011).

4. P. Kramer, A. K. Sharma, E. E. Hennecke, and H. Yasuda, J. Polym. Sci.: Polym. Chem. Ed. 22, 475 (1984).

5. D. Wright, B. Rajalingam, S. Selvarasah, M. R. Dokmeci, and A. Khademhossein, Lab Chip 7, 1272 (2007). 
6. E. Meng and Y.-C. Tai, in Proceedings of 18 IEEE International Conference on Micro Electro Mechanical Systems, Miami Beach, FL, USA, 2005 (IEEE, Miami Beach, 2005), p. 568.

7. J. W. Evans, P. A. Thiel, and M. C. Bartelt, Surf. Sci. Rep. 61, 1 (2006).

8. J. G. Amar and F. Family, Thin Solid Films 272, 208 (1996).

9. J. A. Blackman, Phys. A (Amsterdam, Neth.) 220, 85 (1995).

10. J. A. Venables, Philos. Mag. 27, 697 (1973).

11. J. A. Venables, G. D. T. Spiller, and M. Hanbücken, in Surface Mobilities on Solid Materials (Springer, Boston, 1984), p. 399.

12. J. G. Amar and F. Family, Phys. Rev. Lett. 74, 2066 (1995).

13. A. Pimpinelli, L. Tumbek, and A. Winkler, J. Phys. Chem. Lett. 5 (6), 67 (2014).

14. M. Brinkmann, S. Graff, and F. Biscarini, Phys. Rev. B: Condens. Matter Mater. Phys. 66, 165430 (2002).

15. S. Lorbek, G. Hlawacek, and C. Teichert, Eur. Phys. J.: Appl. Phys 55, 55 (2011).

16. I. J. Lee, M. Yun, S. M. Lee, and J. Y. Kim, Phys. Rev. B: Condens. Matter Mater. Phys. 78, 115427 (2008).

17. I. J. Lee and M. Yun, Macromolecules 43, 5450 (2010).

18. J. G. Amar, F. Family, and P. M. Lam, Phys. Rev. B: Condens. Matter Mater. Phys. 50, 8781 (1994).

19. J. R. Albia and M. A. Albao, Phys. Rev. E 95, 042802 (2017).
20. A. S. Mitko, D. R. Streltsov, P. V. Dmitryakov, A. A. Nesmelov, A. I. Buzin, and S. N. Chvalun, Polym. Sci., Ser. A 61, 555 (2019).

21. W. F. Gorham, J. Polym. Sci., Part A: Polym. Chem. 4, 3027 (1966).

22. Silicon Wafer Cleaning Technology, Ed. by K. A. Reinhardt and W. Kern (William Andrew, New York, 2008), p. 24.

23. Gwyddion. http://gwyddion.net. Cited 2020.

24. A. Choukourov, I. Melnichuk, I. Gordeev, D. Nikitin, R. Tafiichuk, P. Pleskunov, J. Hanuš, J. Houška, T. Kretková, and M. Dopita, Prog. Org. Coat. 143, 105630 (2020).

25. D. L. González, A. Pimpinelli, and T. L. Einstein, Phys. Rev. E 96, 012804 (2017).

26. Y. Han, É. Gaudry, T. J. Oliveira, and J. W. Evans, J. Chem. Phys. 145, 211904 (2016).

27. D. L. González, M. Camargo and J. A. Sánchez, Phys. Rev. E 97, 052802 (2018).

28. J. K. Zuo and J. F. Wendelken, Phys. Rev. Lett. 66, 2227 (1991).

29. J. R. Albia and M. A. Albao, J. Vac. Sci. Technol. 33, 021404 (2015).

30. T. L. Einstein, A. Pimpinelli, and D. Luis González, J. Cryst. Growth 401, 67 (2014).

31. T. L. Einstein, A. Pimpinelli, D. L. González, and J. R. Morales-Cifuentes, J. Phys.: Conf. Ser. 640, 012024 (2015).

32. K. Smalara, A. Giełtdoń, M. Bobrowski, J. Rybicki, and C. Czaplewski, J. Phys. Chem. A 114, 4296 (2010). 